\title{
On vibrations in Green-Naghdi thermoelasticity of dipolar bodies
}

\author{
M. Marin, A. Chirilă, L. Codarcea and S. Vlase
}

\begin{abstract}
This study is concerned with the theory of thermoelasticity of type III proposed by Green and Naghdi, which is extended to cover the bodies with dipolar structure. In this context we construct a boundary value problem for a prismatic bar which is subjected to some harmonic in time vibrations. For the oscillations whose amplitudes have the frequency lower than a critical value, we deduce some estimates for describing the spatial behavior.
\end{abstract}

\section{Introduction}

Many studies published in the last years have highlighted that the classical uncoupled theory of thermoelasticity predicts two phenomena not compatible with concrete experiments: the equation of heat conduction does not contain any elastic terms and the heat equation is of parabolic type and this means that it predicts infinite speeds of propagation for the heat waves. In order to eliminate the paradoxes of the classical theory, a great number of studies were published. In this context, Green and Naghdi developed three different theories, labeled type I, type II and type III, in [1]-[3]. So, the Green-Naghdi theory of type I is equivalent to the classical coupled thermoelasticity theory. In the Green-Naghdi theory of type II the energy of the system is constant in time, in other words, this theory does not admit energy dissipation and

Key Words: dipolar bodies, Green-Naghdi thermoelasticity, G-N type III, harmonic vibrations, spatial decay.

2010 Mathematics Subject Classification: Primary 74A15, 74A35; Secondary 80A17.

Received: 30.01.2018

Accepted: 28.02.2018 
implies a finite speed of propagation for the heat waves. Finally, the GreenNaghdi theory of type III admits dissipation of energy and the heat flux is a combination of type I and type II. Also, this type III implies a finite speed of propagation for the heat waves.

Essentially, the theories of Green-Naghdi are based on an energy balance law rather than an entropy inequality. In the context of thermodynamics the so called "thermal displacement" is introduced, denoted by $\tau$, which is related to the temperature variation $\theta$ by means of the relation

$$
\tau(t)=\int_{0}^{t} \theta(s) d s .
$$

There are other studies (see, for instance, [4]) in which the Fourier law is replaced by an approximation of the equation where the thermal displacement function, the thermal conductivity tensor and the conductivity rate tensor appeared. By using Taylor approximations, it is proved that this theory covers the Green-Naghdi theories. The theory of bodies with microstructure has primarily the aim to remove the differences which occur between experiments and the classical theory of elasticity. The results of classical elasticity prove not to be appropriate when the body's overall deformations are subject to effects of material microstructure. This happens in the case of ceramics, graphite, human bones, polymers (that is, some granular bodies with large molecules), and so on. Eringen was the first to study this kind of theory (see for instance $[5],[6])$ which was continuously studied in various papers, such as [7]-[9]. Some considerations on waves for specific bodies with microstructure can be found in [10]-[13]. A specific aspect of the microstructure is the dipolar structure. Many valuable researchers emphasized the importance of the dipolar structure of materials. The start was given by the published results of R. D. Mindlin [14] as well as A. E. Green and R. S. Rivlin [15], which approached also in other papers the multipolar structures and in particular, the dipolar structures. Another known reseacher, M. E. Gurtin published a few articles on multipolar structures. It is enough to recall the paper [16], where Gurtin together with E. Fried discover integral statements of force balance, energy balance, and entropy imbalance for an interface between a body and its environment. We want to outline that in the theory of dipolar continua the degrees of freedom for each particle are three translations and nine micro-deformations and each material point is constrained to deform homogeneously. The theories of dipolar bodies are quite sufficient for a large number of solid mechanics applications.

Studies on harmonic oscillations appeared long time ago, but have been of great interest for a large number of researchers in the last period of time. So, in the context of the linearly damped wave, Flavin and Knops (1987) treated the spatial behavior of harmonic vibration. Similar results, in other 
conditions, we find in Flavin and Knops (1990) and Knops (1991). Chiriţă in (1996) extended the results to cover the linear theory of thermoelasticity. Also, we must outline the results regarding the wave propagation obtained by Quintanilla and Straughan (2004). We will formulate the mixed initialboundary value problem consisting of equations and conditions in the context described above. These equations and conditions refer to the displacement vector $u_{i}$, the dipolar displacement tensor $\varphi_{i j}$, the temperature $\theta$, the mass density $\varrho$, the thermal capacity $c$, the thermal conductivity tensor, the thermal displacement $\alpha$ and the elastic coefficients tensors. All these quantities are smooth functions of the position, $f=f(x)$. We will see that we need to assume that the heat capacity, the mass density are positive and also, the thermal conductivity tensor and the elasticity tensors are positive definite in order to have the well-posedness of the Green-Naghdi thermoelasticity of type III.

If, on the contrary, we do not assume the positivity of the elasticity tensors, the mixed problem with the usual initial and boundary conditions becomes ill-posed. The plan of our study is the following. In Section 2 we recall the basic notations, the fundamental equations, the initial conditions and the boundary conditions required to define the mixed problem for the type III thermoelasticity of dipolar bodies. In Section 3 we include the main results. In Proposition 1 and Theorem 1 we prove two estimates which are used to obtain the two main results.

\section{Notations and basic equations}

Assume that at time $t_{0}$ our dipolar thermoelastic body occupies the domain $B$ included in the Euclidean three-dimensional space $R^{3}$. Its boundary is the piecewise smooth surface $\partial B$. In $B$ we will use a fixed system of rectangular Cartesian axes $O x_{i}, i=1,2,3$ so that in this system any point $P$ from $\Omega$ is characterized by three rectangular coordinates $x_{1}, x_{2}, x_{3}$ and we use the notation $x$ for $\left(x_{1}, x_{2}, x_{3}\right)$. So, $x$ will be the position and $t$ will be the time. The functions considered in the following are considered to be functions of $(x, t)$ defined on the cylinder $\bar{B} \times(0, \infty)$, where $\bar{B}=B \cup \partial B$. If there is no likelihood of confusion, the spatial variables and the time variable of the functions will be omitted. We will use the known convention of summation over repeated subscripts and differentiation. Greek subscripts are understood to range over the integers $(1,2)$ and Latin subscripts take the values $1,2,3$. We also use a superposed dot to denote the partial differentiation with respect to time, $t, \dot{f}=\partial f / \partial t$, and a subscript preceded by a comma denotes partial differentiation with respect to the corresponding Cartesian coordinate, $f_{, j}=$ $\partial f / \partial x_{j}$. Our mathematical model requires a system of governing equations 
in the context of the linear theory of dipolar thermoelasticity. By using the known procedure of Green and Rivlin we consider a new motion which differs from the given motion only by a superposed rigid motion defined by a rotation of uniform rigid body angular velocity and suppose that for the given motion, all characteristics of the body are unaltered by such a superposed rigid motion. So we deduce the following kinetic relations, which give the expressions of the strain measures $\varepsilon_{i j}, \gamma_{i j}$ and $\chi_{i j k}$ and of the thermal displacement gradient $\alpha_{i}$ with regard to the variables of motion (see Eringen [5])

$$
\varepsilon_{i j}=\frac{1}{2}\left(u_{i, j}+u_{j, i}\right), \gamma_{i j}=u_{j, i}-\varphi_{i j}, \chi_{i j k}=\varphi_{j k, i}, \alpha_{i}=\tau_{, i} .
$$

The motion of the dipolar body in type III thermoelastodynamics will be characterized by the displacement vector of components $\left(u_{i}\right)$, the dipolar displacement tensor of components $\left(\varphi_{i j}\right)$ and the thermal displacement $\alpha$.

We will consider that all components of the displacement and the temperature variation from some reference temperature are small. Also, the space derivatives of these functions and their time derivatives are small.

We restrict our considerations to the case where the materials have a center of symmetry. Also, we suppose that the body is free from stress, in its reference configuration, and has zero intrinsic equilibrated body forces and body couples. The linear theory requires a quadratic form for the specific Helmholtz free energy $H$ with regards to its independent constitutive variables.

$$
\begin{array}{r}
\varrho H=\frac{1}{2} C_{i j m n} \varepsilon_{i j} \varepsilon_{m n}+G_{i j m n} \varepsilon_{i j} \gamma_{m n}+F_{i j m n r} \varepsilon_{i j} \chi_{m n r}+\frac{1}{2} B_{i j m n} \gamma_{i j} \gamma_{m n} \\
+D_{i j m n r} \gamma_{i j} \chi_{m n r}+\frac{1}{2} A_{i j k m n r} \chi_{i j k} \chi_{m n r}+M_{i j m} \varepsilon_{i j} \alpha_{m}+N_{i j m} \gamma_{i j} \alpha_{m} \\
+R_{i j} \alpha_{i} \dot{\alpha}_{i}+P_{i j k m} \chi_{i j k} \alpha_{m}+Q_{i} \alpha_{i} \theta+\frac{1}{2} K_{i j} \alpha_{i} \alpha_{j}-a_{i j} \varepsilon_{i j} \theta \\
-b_{i j} \gamma_{i j} \theta-c_{i j k} \chi_{i j k} \theta-\frac{1}{2} c \theta^{2} .
\end{array}
$$

Correspondingly, the internal energy density $\mathcal{E}$ has the expression

$$
\begin{aligned}
\varrho \mathcal{E}=\frac{1}{2} C_{i j m n} \varepsilon_{i j} \varepsilon_{m n}+ & G_{i j m n} \varepsilon_{i j} \gamma_{m n}+F_{i j m n r} \varepsilon_{i j} \chi_{m n r}+\frac{1}{2} B_{i j m n} \gamma_{i j} \gamma_{m n} \\
+D_{i j m n r} \gamma_{i j} \chi_{m n r}+ & \frac{1}{2} A_{i j k m n r} \chi_{i j k} \chi_{m n r}+M_{i j m} \varepsilon_{i j} \alpha_{m}+N_{i j m} \gamma_{i j} \alpha_{m} \\
& +P_{i j k m} \chi_{i j k} \alpha_{m}+\frac{1}{2} K_{i j} \alpha_{i} \alpha_{j}+R_{i j} \alpha_{i} \dot{\alpha}_{j}+\frac{1}{2} c \theta^{2} .
\end{aligned}
$$

The specific Helmholtz free energy $H$ is used in the inequality of entropy to obtain the constitutive equations that give the expressions for the stress 
measures in terms of the strain measures

$$
\begin{aligned}
& \tau_{i j}=C_{i j m n} \varepsilon_{m n}+G_{m n i j} \gamma_{m n}+F_{m n r i j} \chi_{m n r}-a_{i j} \theta, \\
& \sigma_{i j}=G_{i j m n} \varepsilon_{m n}+B_{i j m n} \gamma_{m n}+D_{i j m n r} \chi_{m n r}-b_{i j} \theta, \\
& \mu_{i j k}=F_{i j k m n} \varepsilon_{m n}+D_{m n i j k} \gamma_{m n}+A_{i j k m n r} \chi_{m n r}-c_{i j k} \theta, \\
& \varrho \eta=a_{i j} \varepsilon_{i j}+b_{i j} \gamma_{i j}+c_{i j k} \chi_{i j k}-N_{i} \alpha_{i}+c \theta, \\
& \quad q_{m}=M_{i j m} \varepsilon_{i j}+N_{i j m} \gamma_{i j}+P_{i j k m} \chi_{i j k}+K_{i m} \alpha_{i}+R_{i m} \dot{\alpha}_{i}+N_{m} \theta .
\end{aligned}
$$

In a similar manner, the motion equations are obtained in the form that follows

$$
\begin{aligned}
& \left(\tau_{i j}+\sigma_{i j}\right)_{, j}+\varrho f_{i}=\varrho \ddot{u}_{i} \\
& \mu_{i j k, i}+\sigma_{j k}+\varrho g_{j k}=I_{j s} \ddot{\varphi}_{k s} .
\end{aligned}
$$

and, also, the energy equation

$$
\varrho \dot{\eta}=q_{i, i}+\varrho r
$$

We must specify that the above equations (1), (5)-(7) take place for $(x, t) \in$ $\bar{B} \times(0, \infty)$.

Also, the notations used in the previous relations have the following meanings: $\varrho$ is the mass density; $I_{j k}$ are the components of the inertia; $\theta$ is the variation of the temperature related to the uniform reference temperature $\theta_{0} ; \varepsilon_{i j}, \gamma_{i j}$, $\chi_{i j k}$ and $\alpha_{i}$ are the strain measures; $\tau_{i j}, \sigma_{i j}$ and $\mu_{i j k}$ are the strain measures; $f_{i}$ are the components of the external body forces; $g_{j k}$ are the components of the external dipolar body forces; $\eta$ is the entropy per unit volume; the components of the heat flux are $q_{i} ; r$ is the external rate of supply of heat per unit mass; $C_{i j m n}, G_{i j m n}, \ldots, Q_{i}$ are the constitutive coefficients and these characteristics of the material, together with the mass density and the heat capacity $c$, are continuously differentiable functions, depending on the spatial variable only and satisfying the following symmetry relations

$$
\begin{gathered}
C_{i j m n}=C_{m n i j}=C_{j i m n}, G_{i j m n}=G_{j i m n}, F_{i j m n r}=F_{j i m n r}, \\
B_{i j m n}=B_{m n i j}, A_{i j k m n r}=A_{m n r i j k}, M_{i j k}=M_{j i k}, K_{i j}=K_{j i} .
\end{gathered}
$$

According to Green and Naghdi [3], we can define the internal rate of supply of the heat per unit mass through the thermal displacement gradient $\alpha_{i}$ as follows

$$
\theta \xi=R_{i j} \dot{\alpha}_{i} \dot{\alpha}_{j}
$$

where the heat conductivity tensor $R_{i j}$ is symmetric and satisfies the dissipation inequality

$$
R_{i j} \dot{\alpha}_{i} \dot{\alpha}_{j} \geq 0
$$




\section{Main results}

In the following we will suppose that the lateral surface of the prismatic bar and its base $x_{3}=h$ are maintained at zero thermal displacement, null displacement and null dipolar displacement and is subject to a given harmonic in time vibration on the base $x_{3}=0$. As a consequence, within the prismatic bar we expect to get a solution that is harmonic in time, that is of the form

$$
u_{j}(x, t)=U_{j}(x) e^{i w t}, \varphi_{j k}(x, t)=\Phi_{j k}(x) e^{i w t}, \tau(x, t)=T(x) e^{i w t},
$$

where $i$ is the complex unit, $i^{2}=-1$, and $w$ is a given positive constant.

If we take into account equations (11), then the kinetic relations (2) become

$$
E_{m n}=\frac{1}{2}\left(U_{m, n}+U_{n, m}\right), \Gamma_{m n}=U_{j, i}-\Phi_{i j}, C_{i j k}=\Phi_{j k, i}, A_{k}=T_{, k} .
$$

As a consequence, the constitutive equations (5) receive the form

$$
\begin{aligned}
& T_{i j}=C_{i j m n} E_{m n}+G_{m n i j} \Gamma_{m n}+F_{m n r i j} C_{m n r}-i w a_{i j} T, \\
& S_{i j}=G_{i j m n} E_{m n}+B_{i j m n} \Gamma_{m n}+D_{i j m n r} C_{m n r}-i w b_{i j} T, \\
& \Lambda_{i j k}=F_{i j k m n} E_{m n}+D_{m n i j k} \Gamma_{m n}+A_{i j k m n r} C_{m n r}-i w c_{i j k} T, \\
& \varrho \eta=a_{i j} E_{i j}+b_{i j} \Gamma_{i j}+c_{i j k} C_{i j k}-N_{i} A_{i}+i w c T \\
& \quad Q_{m}=M_{i j m} E_{i j}+N_{i j m} \Gamma_{i j}+P_{i j k m} C_{i j k}+K_{i m} A_{i}+i w R_{j m} A_{j}+i w N_{m} T .
\end{aligned}
$$

In the absence of the external body forces and of the external dipolar body forces, and considering equations (11) the equations of motion (6) become

$$
\begin{gathered}
\left(T_{m n}+S_{m n}\right)_{, n}+\varrho w^{2} U_{m}=0, \\
\Lambda_{k m n, n}+S_{k m}+w^{2} I_{k s} \Phi_{m s}=0 .
\end{gathered}
$$

If we take into account equations (11), the energy equation (7) receives the form

$$
Q_{j, j}-i w a_{j k} E_{j k}-i w b_{j k} \Gamma_{j k}-i w c_{j k m} C_{j k m}+i w N_{m} A_{m}+c w^{2} T=0 .
$$

Along with the basic equations above, we will now add the boundary conditions. The generic cross-section of the cylinder will be denoted by $\Omega$ and assume that its boundary $\partial \Omega$ is smooth enough to apply the divergence theorem. Then the boundary conditions on the base of the cylinder, that is, the end $x_{3}=0$ have the form

$$
U_{j}(x)=\tilde{U}_{j}(x), \Phi_{j k}(x)=\tilde{\Phi}_{j k}(x), T(x)=\tilde{T}(x) \text {, on } \Omega(0) .
$$


On the end $x_{3}=l$ we have

$$
U_{j}(x)=0, \Phi_{j k}(x)=0, T(x)=0, \text { on } \Omega(l),
$$

where $l$ is the length of the cylinder.

The lateral boundary conditions have a similar form

$$
U_{j}(x)=0, \Phi_{j k}(x)=0, T(x)=0, \text { on } \partial \Omega \times[0, l] .
$$

We will denote with $(P)$ the boundary problem that consists of the basic equations (12)-(15) and the boundary conditions (16)-(18).

The following two estimates will be useful in the following.

Theorem 1.. If $\left(U_{j}, \Phi_{j k}, T\right)$ is the amplitude of a vibration which is a solution of the problem $(P)$, then the following two estimates take place

$$
\begin{array}{r}
2 \int_{\Omega\left(x_{3}\right)}\left[C_{k l m n} E_{k l} \bar{E}_{m n}+G_{k l m n}\left(E_{k l} \bar{\Gamma}_{m n}+\bar{E}_{k l} \Gamma_{m n}\right)+F_{k l m n r}\left(E_{k l} \bar{C}_{m n r}\right.\right. \\
\left.+\bar{E}_{k l} C_{m n r}\right)+B_{k l m n} \Gamma_{k l} \bar{\Gamma}_{m n}+D_{k l m n r}\left(\Gamma_{k l} \bar{C}_{m n r}+\bar{\Gamma}_{k l} C_{m n r}\right) \\
+A_{j k l m n r} C_{j k l} \bar{C}_{m n r}+M_{k l m}\left(E_{k l} \bar{A}_{m}+\bar{E}_{k l} A_{m}\right)+N_{k l m}\left(\Gamma_{k l} \bar{A}_{m}+\bar{\Gamma}_{k l} A_{m}\right) \\
+P_{j k l m}\left(C_{j k l} \bar{A}_{m}+\bar{C}_{j k l} A_{m}\right)+K_{j l} \alpha_{k} \bar{A}_{j}-w^{2}\left(\varrho U_{j} \bar{U}_{j}+I_{m n} \Phi_{m k} \bar{\Phi}_{n k}\right. \\
+c T \bar{T})+i w a_{j k}\left(E_{j k} \bar{T}-\bar{E}_{j k} T\right)+i w b_{j k}\left(\Gamma_{j k} \bar{T}_{-} \bar{\Gamma}_{j k} T\right) \\
\left.+i w c_{j k m}\left(C_{j k m} \bar{T}-\bar{C}_{j k m} T\right)+i w N_{m}\left(T \bar{T}_{, m}-\bar{T} T_{, m}\right)\right] d A \\
=\frac{d}{d x_{3}} \int_{\Omega\left(x_{3}\right)}\left[U_{m}\left(\bar{S}_{3 m}+\bar{T}_{3 m}\right)+\bar{U}_{m}\left(S_{3 m}+T_{3 m}\right)\right. \\
\left.+\Phi_{j k} \bar{\Lambda}_{3 j k}+\bar{\Phi}_{j k} \Lambda_{3 j k}+\left(T \bar{Q}_{3}+\bar{T} Q_{3}\right)\right] d A \\
+\frac{d}{d x_{3} \int_{\Omega\left(x_{3}\right)} \frac{i}{2 w}\left[U_{m}\left(\bar{S}_{3 m}+\bar{T}_{3 m}\right)+\Phi_{j k} \bar{\Lambda}_{3 j k}-\bar{U}_{m}\left(S_{3 m}+T_{3 m}\right)-\bar{\Phi}_{j k} \Lambda_{3 j k}\right] d A} \\
\int_{\Omega\left(x_{3}\right)} R_{m n} A_{m} \bar{A}_{n} d A=\frac{i}{2 w}\left(T \bar{Q}_{3}-\bar{T}_{3}\right) d A_{\Omega\left(x_{3}\right)}
\end{array}
$$

where a bar over a function refers to the complex conjugate of the respective function.

Proof. We multiply in $(13)_{1}$ and $(13)_{2}$ by $\bar{U}_{n}$, in $(13)_{3}$ by $\bar{\Phi}_{n r}$ and in $(13)_{5}$ by $\bar{T}$, then we add the three relations that result. If we use the equations (14) 
and (15) we are led to the relation

$$
\begin{array}{r}
{\left[\left(T_{m n}+S_{m n}\right) \bar{U}_{n}+\Lambda_{m n r} \bar{\Phi}_{n r}+Q_{m} \bar{T}\right]_{, m}} \\
=C_{k l m n} E_{k l} \bar{E}_{m n}+G_{k l m n}\left(E_{k l} \bar{\Gamma}_{m n}+\bar{E}_{k l} \Gamma_{m n}\right)+F_{k l m n r}\left(E_{k l} \bar{C}_{m n r}\right. \\
\left.+\bar{E}_{k l} C_{m n r}\right)+B_{k l m n} \Gamma_{k l} \bar{\Gamma}_{m n}+D_{k l m n r}\left(\Gamma_{k l} \bar{C}_{m n r}+\bar{\Gamma}_{k l} C_{m n r}\right) \\
+A_{j k l m n r} C_{j k l} \bar{C}_{m n r}+M_{k l m}\left(E_{k l} \bar{A}_{m}+\bar{E}_{k l} A_{m}\right)+N_{k l m}\left(\Gamma_{k l} \bar{A}_{m}+\bar{\Gamma}_{k l} A_{m}\right) \\
+P_{j k l m}\left(C_{j k l} \bar{A}_{m}+\bar{C}_{j k l} A_{m}\right)+K_{j l} A_{l} \bar{A}_{j}-w^{2}\left(\varrho U_{j} \bar{U}_{j}+I_{m n} \Phi_{m k} \bar{\Phi}_{n k}\right. \\
+c T \bar{T})+i w a_{j k}\left(E_{j k} \bar{T}-\bar{E}_{j k} T\right)+i w b_{j k}\left(\Gamma_{j k} \bar{T}_{-} \bar{\Gamma}_{j k} T\right) \\
+i w c_{j k m}\left(C_{j k m} \bar{T}-\bar{C}_{j k m} T\right)+i w N_{m}\left(T \bar{T}_{, m}-\bar{T} T_{, m}\right)+i w R_{m n} A_{m} \bar{A}_{n} .
\end{array}
$$

Now we apply the complex conjugate in $(13)_{1}-(13)_{3}$ and $(13)_{5}$ and then in the first relation that we obtain, we multiply by $U_{n}$, in the second by $\Phi_{n r}$ and in the last by $T$. If we add the three relations and use the equations (14) and (15), we obtain the relation

$$
\begin{array}{r}
{\left[\left(\bar{T}_{m n}+\bar{S}_{m n}\right) U_{n}+\bar{\Lambda}_{m n r} \Phi_{n r}+\bar{Q}_{m} T\right]_{, m}} \\
=C_{k l m n} E_{k l} \bar{E}_{m n}+G_{k l m n}\left(E_{k l} \bar{\Gamma}_{m n}+\bar{E}_{k l} \Gamma_{m n}\right)+F_{k l m n r}\left(E_{k l} \bar{C}_{m n r}\right. \\
\left.+\bar{E}_{k l} C_{m n r}\right)+B_{k l m n} \Gamma_{k l} \bar{\Gamma}_{m n}+D_{k l m n r}\left(\Gamma_{k l} \bar{C}_{m n r}+\bar{\Gamma}_{k l} C_{m n r}\right) \\
+A_{j k l m n r} C_{j k l} \bar{C}_{m n r}+M_{k l m}\left(E_{k l} \bar{A}_{m}+\bar{E}_{k l} A_{m}\right)+N_{k l m}\left(\Gamma_{k l} \bar{A}_{m}+\bar{\Gamma}_{k l} A_{m}\right) \\
+P_{j k l m}\left(C_{j k l} \bar{A}_{m}+\bar{C}_{j k l} A_{m}\right)+K_{j l} A_{l} \bar{A}_{j}-w^{2}\left(\varrho U_{j} \bar{U}_{j}+I_{m n} \Phi_{m k} \bar{\Phi}_{n k}\right. \\
+c T \bar{T})+i w a_{j k}\left(E_{j k} \bar{T}-\bar{E}_{j k} T\right)+i w b_{j k}\left(\Gamma_{j k} \bar{T}_{-} \bar{\Gamma}_{j k} T\right) \\
+i w c_{j k m}\left(C_{j k m} \bar{T}-\bar{C}_{j k m} T\right)+i w N_{m}\left(T \bar{T}_{, m}-\bar{T} T_{, m}\right)-i w R_{m n} A_{m} \bar{A}_{n},
\end{array}
$$

in which we took into account the symmetry relations (8).

Finally, we integrate in $(21)$ and $(22)$ on the cross-section $\Omega\left(x_{3}\right)$ and with the help of the lateral boundary conditions (18) we get the desired relations (19) and (20).

In order to obtain our main result, namely a spatial behavior of the amplitude $\left(U_{j}, \Phi_{j k}, T\right)$ of the harmonic vibrations which is a solution of the problem $(P)$, we need the following function

$$
\begin{gathered}
\mathcal{F}\left(x_{3}\right)=-\frac{\delta i}{2 w} \int_{\Omega\left(x_{3}\right)}\left[U_{m}\left(\bar{S}_{3 m}+\bar{T}_{3 m}\right)+\Phi_{j k} \bar{\Lambda}_{3 j k}-\bar{U}_{m}\left(S_{3 m}+T_{3 m}\right)\right. \\
\left.-\bar{\Phi}_{j k} \Lambda_{3 j k}\right] d A-\frac{1}{2} \int_{\Omega\left(x_{3}\right)}\left[U_{m}\left(\bar{S}_{3 m}+\bar{T}_{3 m}\right)+\Phi_{j k} \bar{\Lambda}_{3 j k}+\bar{U}_{m}\left(S_{3 m}+T_{3 m}\right)\right. \\
\left.+\bar{\Phi}_{j k} \Lambda_{3 j k}\right] d A+\frac{1}{2} \int_{\Omega\left(x_{3}\right)}\left[\frac{\delta i}{w}\left(T \bar{Q}_{3}-\bar{T} Q_{3}\right)+\left(T \bar{Q}_{3}+\bar{T} Q_{3}\right)\right] d A
\end{gathered}
$$


where $x_{3} \geq 0$ and $\delta$ is a parameter which can take any positive value we need. Also, in order to estimate the amplitude of the harmonic vibration, we need the following measure

$$
\begin{aligned}
\mathcal{M}\left(x_{3}\right) & =\lambda_{1} \int_{\Omega\left(x_{3}\right)} C_{k l m n} E_{k l} \bar{E}_{m n} d V+\lambda_{2} \int_{\Omega\left(x_{3}\right)} B_{k l m n} \Gamma_{k l} \bar{\Gamma}_{m n} d V \\
& +\lambda_{3} \int_{\Omega\left(x_{3}\right)} A_{j k l m n r} C_{j k l} \bar{C}_{m n r} d V+\lambda_{4} \int_{\Omega\left(x_{3}\right)} R_{m n} A_{m} \bar{A}_{n} d V
\end{aligned}
$$

where $\lambda_{1}, \lambda_{2}, \lambda_{3}$ and $\lambda_{4}$ are positive parameters which can be conveniently chosen

We will use the following hypotheses:

i) the mass density and the tensor of inertia are strictly positive, that is, $\varrho>0, I_{j k}>0$

ii) the heat conductivity tensor $R_{m n}$ satisfies the condition:

$$
\mu_{m} \xi_{m} \xi_{m} \leq R_{m n} \xi_{m} \xi_{n} \leq \mu_{M} \xi_{m} \xi_{m} \text { for all } \xi_{m}
$$

where the positive constants $\mu_{m}$ and $\mu_{M}$ are related to the minimum and maximum eigenvalues of the positive definite tensor $R_{m n}$.

Similar conditions satisfy the elasticity tensors that occur in the internal energy density.

Theorem 2.. If $\left(U_{j}, \Phi_{j k}, T\right)$ is the amplitude of a vibration which is a solution of the problem $(P)$ having a frequency $w$ lower than a prescribed value $w^{*}$, then we can compute a constant $\nu$ such that the measure $\mathcal{M}\left(x_{3}\right)$ satisfies the following estimate

$$
0 \leq \mathcal{M}\left(x_{3}\right) \leq \mathcal{M}(0) e^{\nu\left(h-x_{3}\right)}, h \leq x_{3} \leq l, h \in(0, l] .
$$

Proof. Let us denote by $\lambda_{0}$ the first eigenvalue of the problem

$$
\begin{aligned}
& u, \beta \beta=-\lambda u \text { in } \Omega, \\
& u=0 \text { on } \partial \Omega .
\end{aligned}
$$

Clearly, (27) is the clamped membrane problem.

Based on the lateral boundary conditions (18) we can deduce that

$$
\int_{\Omega\left(x_{3}\right)} T_{, \beta} \bar{T}_{, \beta} d A \geq \int_{\Omega\left(x_{3}\right)} T \bar{T} d A .
$$


By using the relations (19) and (20) we are led to

$$
\begin{array}{r}
\frac{d \mathcal{F}\left(x_{3}\right)}{d x_{3}} \\
=-\int_{\Omega\left(x_{3}\right)}\left[\delta R_{m n} A_{m} \bar{A}_{n}+C_{k l m n} E_{k l} \bar{E}_{m n}+G_{k l m n}\left(E_{k l} \bar{\Gamma}_{m n}+\bar{E}_{k l} \Gamma_{m n}\right)\right. \\
+F_{k l m n r}\left(E_{k l} \bar{C}_{m n r}+\bar{E}_{k l} C_{m n r}\right)+D_{k l m n r}\left(\Gamma_{k l} \bar{C}_{m n r}+\bar{\Gamma}_{k l} C_{m n r}\right) \\
+B_{k l m n} \Gamma_{k l} \bar{\Gamma}_{m n}+A_{j k l m n r} C_{j k l} \bar{C}_{m n r}+M_{k l m}\left(E_{k l} \bar{A}_{m}+\bar{E}_{k l} A_{m}\right) \\
+N_{k l m}\left(\Gamma_{k l} \bar{A}_{m}+\bar{\Gamma}_{k l} A_{m}\right)+P_{j k l m}\left(C_{j k l} \bar{A}_{m}+\bar{C}_{j k l} A_{m}\right)+K_{j l} A_{k} \bar{A}_{l} \\
-w^{2}\left(\varrho U_{j} \bar{U}_{j}+I_{m n} \Phi_{m k} \bar{\Phi}_{n k}+c T \bar{T}\right)+i w a_{j k}\left(E_{j k} \bar{T}-\bar{E}_{j k} T\right) \\
+i w b_{j k}\left(\Gamma_{j k} \bar{T}-\bar{\Gamma}_{j k} T\right)+i w c_{j k m}\left(C_{j k m} \bar{T}-\bar{C}_{j k m} T\right) \\
\left.+i w N_{m}\left(T \bar{T}_{, m}-\bar{T} T_{, m}\right)\right] d A, \text { for any } x_{3} \geq 0 .
\end{array}
$$

By using the Cauchy-Schwarz inequality and also the arithmetic-geometric mean inequality, we can obtain some upper bounds for the integrals in the right-hand side of equation (29). For instance, if we use the notation

$$
M^{*}=\sup _{B} \sqrt{M_{k l m} M_{k l m}}
$$

we have

$$
\begin{aligned}
\left|\int_{\Omega\left(x_{3}\right)} M_{k l m}\left(E_{k l} \bar{A}_{m}+\bar{E}_{k l} A_{m}\right) d A\right| & \leq \frac{M^{*}}{\mu_{m}} p_{1} \int_{\Omega\left(x_{3}\right)} C_{k l m n} E_{k l} \bar{E}_{m n} d A \\
+ & \frac{M^{*}}{\nu p_{1}} \int_{\Omega\left(x_{3}\right)} R_{m n} A_{m} \bar{A}_{n} d A, \forall p_{1}>0 .
\end{aligned}
$$

Also, we have

$$
\begin{aligned}
&\left|\int_{\Omega\left(x_{3}\right)} N_{k l m}\left(\Gamma_{k l} \bar{A}_{m}+\bar{\Gamma}_{k l} A_{m}\right) d A\right| \leq \frac{N^{*}}{\mu_{m}} p_{2} \int_{\Omega\left(x_{3}\right)} B_{k l m n} \Gamma_{k l} \bar{\Gamma}_{m n} d A \\
&+\frac{N^{*}}{\nu p_{2}} \int_{\Omega\left(x_{3}\right)} R_{m n} A_{m} \bar{A}_{n} d A, \forall p_{2}>0, \\
&\left|\int_{\Omega\left(x_{3}\right)} P_{j k l m}\left(C_{j k l} \bar{A}_{m}+\bar{C}_{j k l} A_{m}\right) d A\right| \leq \frac{P^{*}}{\mu_{m}} p_{3} \int_{\Omega\left(x_{3}\right)} A_{j k l m n r} C_{j k l} \bar{C}_{m n r} d A \\
&+\frac{P^{*}}{\nu p_{3}} \int_{\Omega\left(x_{3}\right)} R_{m n} A_{m} \bar{A}_{n} d A, \forall p_{3}>0,
\end{aligned}
$$


where

$$
N^{*}=\sup _{B} \sqrt{N_{k l m} N_{k l m}}, \quad P^{*}=\sup _{B} \sqrt{P_{j k l m} P_{j k l m}} .
$$

For the other integrals from (29) we deduce

$$
\begin{aligned}
& \left|\int_{\Omega\left(x_{3}\right)} K_{m n} A_{m} \bar{A}_{n} d A\right| \leq \frac{K^{*}}{\mu_{m}} \int_{\Omega\left(x_{3}\right)} R_{m n} A_{m} \bar{A}_{n} d A \\
& K^{*}=\sup _{B} \sqrt{K_{m n} K_{m n}}, \\
& \left|\int_{\Omega\left(x_{3}\right)} c w^{2} T \bar{T} d A\right| \leq w^{2} c^{*} \int_{\Omega\left(x_{3}\right)} T \bar{T} d A \\
& \leq \frac{w^{2} c^{*}}{\lambda_{0} \mu_{m}} \int_{\Omega\left(x_{3}\right)} R_{m n} A_{m} \bar{A}_{n} d A, \quad c^{*}=\sup _{B}|c|, \\
& \left|\int_{\Omega\left(x_{3}\right)} i w a_{j k}\left(E_{j k} \bar{T}-\bar{E}_{j k} T\right) d A\right| \leq \frac{w a^{*} p_{4}}{\mu_{m}} \int_{\Omega\left(x_{3}\right)} C_{k l m n} E_{k l} \bar{E}_{m n} d A \\
& +\frac{w a^{*}}{\lambda_{0} \nu_{m} p_{4}} \int_{\Omega\left(x_{3}\right)} R_{m n} A_{m} \bar{A}_{n} d A, p_{4}>0, a^{*}=\sup _{B} \sqrt{a_{m n} a_{m n}}, \\
& \left|\int_{\Omega\left(x_{3}\right)} i w b_{j k}\left(\Gamma_{j k} \bar{T}-\bar{\Gamma}_{j k} T\right) d A\right| \leq \frac{w b^{*} p_{5}}{\mu_{m}} \int_{\Omega\left(x_{3}\right)} B_{k l m n} \Gamma_{k l} \Gamma E_{m n} d A \\
& +\frac{w b^{*}}{\lambda_{0} \nu_{m} p_{5}} \int_{\Omega\left(x_{3}\right)} R_{m n} A_{m} \bar{A}_{n} d A, p_{5}>0, b^{*}=\sup _{B} \sqrt{b_{m n} b_{m n}}, \\
& \left|\int_{\Omega\left(x_{3}\right)} i w c_{j k m}\left(C_{j k m} \bar{T}-\bar{C}_{j k m} T\right) d A\right| \leq \frac{w c^{*} p_{6}}{\mu_{m}} \int_{\Omega\left(x_{3}\right)} A_{j k l m n r} C_{j k l} \\
& \cdot \bar{C}_{m n r} d A+\frac{w c^{*}}{\lambda_{0} \nu_{m} p_{6}} \int_{\Omega\left(x_{3}\right)} R_{m n} A_{m} \bar{A}_{n} d A, p_{6}>0, c^{*}=\sup _{B} \sqrt{c_{m n r} c_{m n r}} \\
& \left|\int_{\Omega\left(x_{3}\right)} i w N_{m}\left(T \bar{T}_{, m}-\bar{T} T_{, m}\right) d A\right| \\
& \leq \frac{w N_{1}^{*}}{\nu_{m} \sqrt{\lambda_{0}}} \int_{\Omega\left(x_{3}\right)} R_{m n} A_{m} \bar{A}_{n} d A, N_{1}^{*}=\sup _{B} \sqrt{N_{m} N_{m}}
\end{aligned}
$$




$$
\begin{aligned}
& \left|\int_{\Omega\left(x_{3}\right)} G_{k l m n}\left(E_{k l} \bar{\Gamma}_{m n}+\bar{E}_{k l} \Gamma_{m n}\right) d A\right| \leq \frac{G^{*}}{\mu_{m}} p_{7} \int_{\Omega\left(x_{3}\right)} C_{k l m n} E_{k l} \bar{E}_{m n} d A \\
& +\frac{G^{*}}{\nu p_{7}} \int_{\Omega\left(x_{3}\right)} B_{k l m n} \Gamma_{k l} \bar{\Gamma}_{m n} d A, p_{7}>0, G^{*}=\sup _{B} \sqrt{G_{k l m n} G_{k l m n}} \\
& \left|\int_{\Omega\left(x_{3}\right)} F_{k l m n r}\left(E_{k l} \bar{C}_{m n r}+\bar{E}_{k l} C_{m n r}\right) d A\right| \\
& \leq \frac{F^{*}}{\mu_{m}} p_{8} \int_{\Omega\left(x_{3}\right)} C_{k l m n} E_{k l} \bar{E}_{m n} d A+\frac{F^{*}}{\nu p_{8}} \int_{\Omega\left(x_{3}\right)} A_{j k l m n r} C_{j k l} \bar{C}_{m n r} d A, \\
& p_{8}>0, F^{*}=\sup _{B} \sqrt{F_{k l m n r} F_{k l m n r}}, \\
& \left|\int_{\Omega\left(x_{3}\right)} D_{k l m n r}\left(\Gamma_{k l} \bar{C}_{m n r}+\bar{\Gamma}_{k l} C_{m n r}\right) d A\right| \leq \frac{D^{*}}{\mu_{m}} p_{9} \int_{\Omega\left(x_{3}\right)} B_{k l m n} \Gamma_{k l} \bar{\Gamma}_{m n} d A \\
& +\frac{D^{*}}{\nu p_{9}} \int_{\Omega\left(x_{3}\right)} A_{j k l m n r} C_{j k l} \bar{C}_{m n r} d A, p_{9}>0, D^{*}=\sup _{B} \sqrt{D_{k l m n r} D_{k l m n r}}
\end{aligned}
$$

If we take into account the estimates (30)-(41), from (29) we are led to the inequality

$$
\begin{aligned}
&-\frac{d \mathcal{F}\left(x_{3}\right)}{d x_{3}} \geq\left(1-\frac{M^{*} p_{1}}{\mu_{m}}-\frac{w a^{*} p_{4}}{\mu_{m}}-\frac{G^{*} p_{7}}{\mu_{m}}-\frac{F^{*} p_{8}}{\mu_{m}}\right) \int_{\Omega\left(x_{3}\right)} C_{k l m n} E_{k l} \bar{E}_{m n} d A \\
&+\left(1-\frac{N^{*} p_{2}}{\mu_{m}}-\frac{w b^{*} p_{5}}{\mu_{m}}-\frac{G^{*}}{\nu p_{7}}-\frac{D^{*} p_{9}}{\mu_{m}}\right) \int_{\Omega\left(x_{3}\right)} B_{k l m n} \Gamma_{k l} \bar{\Gamma}_{m n} d A \\
&+\left(1-\frac{P^{*} p_{3}}{\mu_{m}}-\frac{w c^{*} p_{6}}{\mu_{m}}-\frac{F^{*}}{\nu p_{8}}-\frac{D^{*}}{\nu p_{9}}\right) \int_{\Omega\left(x_{3}\right)} A_{j k l m n r} C_{j k l} \bar{C}_{m n r} d A \\
&+\left(\delta-\frac{M^{*}}{\nu p_{1}}+\frac{N^{*}}{\nu p_{2}}+\frac{P^{*}}{\nu p_{3}}-\frac{K^{*}}{\mu_{m}}-\frac{w^{2} c_{1}^{*}}{\lambda_{0} \mu_{m}}-\frac{w a^{*}}{\lambda_{0} \nu_{m} p_{4}}-\frac{w b^{*}}{\lambda_{0} \nu_{m} p_{5}}-\frac{w c^{*}}{\lambda_{0} \nu_{m} p_{6}}-\frac{w N_{1}^{*}}{\sqrt{\lambda_{0}} \nu_{m}}\right) \\
& \times \int_{\Omega\left(x_{3}\right)} R_{m n} A_{m} \bar{A}_{n} d A .
\end{aligned}
$$

According to Flavin and Knops (1987), the frequency of the vibration must satisfy the following restriction

$$
0<w<\inf \frac{\int_{B}\left[C_{k l m n} E_{k l} \bar{E}_{m n}+B_{k l m n} \Gamma_{k l} \bar{\Gamma}_{m n}+A_{j k l m n r} C_{j k l} \bar{C}_{m n r}\right] d V}{\int_{B}\left(\varrho U_{, m} U_{, n}+I_{m n} \Phi_{m k} \bar{\Phi}_{n k}\right) d V}
$$


If we take into account the end boundary conditions (17), from (23) we deduce that $\mathcal{F}(l)=0$.

On the other hand, if we choose

$$
\begin{array}{r}
\lambda_{1}=\left(1-\frac{M^{*} p_{1}}{\mu_{m}}-\frac{w a^{*} p_{4}}{\mu_{m}}-\frac{G^{*} p_{7}}{\mu_{m}}-\frac{F^{*} p_{8}}{\mu_{m}}\right), \\
\lambda_{2}=\left(1-\frac{N^{*} p_{2}}{\mu_{m}}-\frac{w b^{*} p_{5}}{\mu_{m}}-\frac{G^{*}}{\nu p_{7}}-\frac{D^{*} p_{9}}{\mu_{m}}\right), \\
\lambda_{3}=\left(1-\frac{P^{*} p_{3}}{\mu_{m}}-\frac{w c^{*} p_{6}}{\mu_{m}}-\frac{F^{*}}{\nu p_{8}}-\frac{D^{*}}{\nu p_{9}}\right), \\
\lambda_{4}=\left(\delta-\frac{M^{*}}{\nu p_{1}}+\frac{N^{*}}{\nu p_{2}}+\frac{P^{*}}{\nu p_{3}}-\frac{K^{*}}{\mu_{m}}-\frac{w^{2} c_{1}^{*}}{\lambda_{0} \mu_{m}}\right. \\
\left.-\frac{w a^{*}}{\lambda_{0} \nu_{m} p_{4}}-\frac{w b^{*}}{\lambda_{0} \nu_{m} p_{5}}-\frac{w c^{*}}{\lambda_{0} \nu_{m} p_{6}}-\frac{w N_{1}^{*}}{\sqrt{\lambda_{0}} \nu_{m}}\right),
\end{array}
$$

then taking into account equations (42) and (43), from (29) we deduce that

$$
\begin{aligned}
& \mathcal{F}\left(x_{3}\right) \geq \lambda_{1} \int_{\Omega\left(x_{3}\right)} C_{k l m n} E_{k l} \bar{E}_{m n} d V+\lambda_{2} \int_{\Omega\left(x_{3}\right)} B_{k l m n} \Gamma_{k l} \bar{\Gamma}_{m n} d V \\
& \quad+\lambda_{3} \int_{\Omega\left(x_{3}\right)} A_{j k l m n r} C_{j k l} \bar{C}_{m n r} d V+\lambda_{4} \int_{\Omega\left(x_{3}\right)} R_{m n} A_{m} \bar{A}_{n} d V .
\end{aligned}
$$

Let us introduce the notation

$$
\Omega\left(x_{3}, h\right)=\Omega\left(x_{3}\right) \backslash \Omega\left(x_{3}+h\right) .
$$

Then, using (24) we deduce that

$$
\begin{array}{r}
-\frac{\partial}{\partial x_{3}}\left(\frac{1}{h} \int_{x_{3}}^{x_{3}+h} \mathcal{M}(\tau) d \tau\right)=-\frac{1}{h}\left[\mathcal{M}\left(x_{3}+h\right)-\mathcal{M}\left(x_{3}\right)\right] \\
=-\frac{1}{h} \int_{x_{3}}^{x_{3}+h} \frac{\partial \mathcal{M}}{\partial \tau}(\tau) d \tau=\frac{\lambda_{1}}{h} \int_{\Omega\left(x_{3}\right)} C_{k l m n} E_{k l} \bar{E}_{m n} d V+\frac{\lambda_{2}}{h} \int_{\Omega\left(x_{3}\right)} B_{k l m n} \Gamma_{k l} \\
\cdot \bar{\Gamma}_{m n} d V+\frac{\lambda_{3}}{h} \int_{\Omega\left(x_{3}\right)} A_{j k l m n r} C_{j k l} \bar{C}_{m n r} d V+\frac{\lambda_{4}}{h} \int_{\Omega\left(x_{3}\right)} R_{m n} A_{m} \bar{A}_{n} d V .
\end{array}
$$

Using the definitions (23) and (24) and the inequality (45), we are led to

$$
\begin{array}{r}
0 \leq \mathcal{M}\left(x_{3}\right) \leq \mathcal{F}\left(x_{3}\right) \leq \frac{w+\delta}{2 w} \int_{\Omega\left(x_{3}\right)}\left|T \bar{Q}_{3}+\bar{T} Q_{3}\right| d A \\
+\frac{w+\delta}{2 w} \int_{\Omega\left(x_{3}\right)}\left[U_{m}\left(\bar{S}_{3 m}+\bar{T}_{3 m}\right)+\Phi_{j k} \bar{\Lambda}_{3 j k}+\bar{U}_{m}\left(S_{3 m}+T_{3 m}\right)+\bar{\Phi}_{j k} \Lambda_{3 j k}\right] d A
\end{array}
$$


from where we deduce that we can calculate the positive constants $\nu_{1}, \nu_{2}, \nu_{3}$ and $\nu_{4}$ so that

$$
\begin{aligned}
& 0 \leq \frac{1}{h} \int_{x_{3}}^{x_{3}+h} \mathcal{M}(\tau) d \tau \leq \frac{\nu_{1}}{h} \int_{\Omega\left(x_{3}\right)} C_{k l m n} E_{k l} \bar{E}_{m n} d V \\
& +\frac{\nu_{2}}{h} \int_{\Omega\left(x_{3}\right)} B_{k l m n} \Gamma_{k l} \bar{\Gamma}_{m n} d V+\frac{\nu_{3}}{h} \int_{\Omega\left(x_{3}\right)} A_{j k l m n r} C_{j k l} \bar{C}_{m n r} d V \\
& +\frac{\nu_{4}}{h} \int_{\Omega\left(x_{3}\right)} R_{m n} A_{m} \bar{A}_{n} d V .
\end{aligned}
$$

Now we introduce the decay rate $\chi$ by

$$
\frac{1}{\chi}=\max \left\{\frac{\nu_{1}}{h}, \frac{\nu_{2}}{h}, \frac{\nu_{3}}{h}, \frac{\nu_{1}}{h}\right\}
$$

and from (46) and (48) we obtain the following differential inequality

$$
\frac{\chi}{h} \int_{x_{3}}^{x_{3}+h} \mathcal{M}(\tau) d \tau+\frac{\partial}{\partial x_{3}}\left(\frac{1}{h} \int_{x_{3}}^{x_{3}+h} \mathcal{M}(\tau) d \tau\right) \leq 0,
$$

for any $x_{3} \in[0, l-h]$.

Clearly, by integrating in (49) we obtain the estimate

$$
0 \leq \int_{x_{3}}^{x_{3}+h} \mathcal{M}(\tau) d \tau \leq \int_{0}^{h} \mathcal{M}(\tau) d \tau e^{-i x_{3}} .
$$

But $\mathcal{M}\left(x_{3}\right)$ is a non-increasing function on the interval $[0, l]$, so we can deduce that

$$
\mathcal{M}\left(x_{3}+h\right) \leq \frac{1}{h} \int_{x_{3}}^{x_{3}+h} \mathcal{M}(\tau) d \tau \leq \mathcal{M}\left(x_{3}\right),
$$

and, taking into account this inequality, from (50) we obtain the desired estimate (26) and the proof of the theorem is concluded.

\section{Conclusions}

It should be stressed that the procedure used in this study is slightly different from that used in the classical case of simple elastic solids. Although the context offered by the Green-Naghdi thermoelasticity of type III for dipolar bodies is much more complicated, the estimates describing the spatial behavior of the harmonic vibrations were obtained in a similar manner. This behavior is obtained only for the amplitudes for which the frequency is lower than a 
critical value, which is influenced only by the mechanical effects. It can not be deduced that the choice of $\chi$ as above determines the best value for the decay rate, but surely it assures an exponential decay. It can be anticipated that the results remain valid for other end boundary conditions provided that the heat flux and the tractions are assumed to be harmonic in time functions and provided that the lateral boundary conditions are null.

\section{References}

[1] Green, A.E., Naghdi, P.M., Re-examination of the basic postulates of thermomechanics. Proc. R. Soc. Lond. A, 432 (1991), 1171-1194.

[2] Green, A.E., Naghdi, P.M., On undamped heat wave in elastic solids. J. Thermal Stress. 15(2) (1992), 253-264.

[3] Green, A.E., Naghdi, P.M., Thermoelasticity without energy dissipation. J. Elast. 9 (1993), 1-8.

[4] Choudhuri, S.K.R., On a thermoelastic three-phase-lag model. J. Thermal Stress. 30(3) (2007), 231-238.

[5] Eringen, A.C., Theory of thermo-microstretch elastic solids, Int. J. Engng. Sci., 28 (1990), 1291-1301.

[6] Eringen, A.C., Microcontinuum Field Theories, Springer-Verlag, New York, 1990.

[7] Iesan, D., Ciarletta, M., Non-Classical Elastic Solids, Longman Scientific and Technical, Harlow, Essex, UK and John Wiley \& Sons, Inc., New York, 1993.

[8] Marin, M., Ochsner, A., The effect of a dipolar structure on the Holder stability in Green-Naghdi thermoelasticity, Contin. Mech. Thermodyn., 29(6) (2017), 1365-1374.

[9] Marin, M., Cesaro means in thermoelasticity of dipolar bodies, Acta Mech., 122(1-4) (1997), 155-168.

[10] Marin, M., Stan, G., Weak solutions in Elasticity of dipolar bodies with stretch, Carpathian J. Math., 29(1) (2013), 33-40.

[11] Straughan, B., Heat waves, in: Applied Mathematical Sciences, vol. 177, Springer, New York, 2011.

[12] Marin, M., Abbas I., Carstea, C., On continuous dependence for the mixed problem of microstretch bodies, An. St. Univ. Ovidius Constanta, 25(1) (2017), 131-143.

[13] Marin, M. Weak Solutions in Elasticity of Dipolar Porous Materials, Math Probl Eng 2008, Art. No. 158908 (2008), 1-8.

[14] Mindlin, R.D., Micro-structure in linear elasticity, Arch. Rational Mech. Anal., 16 (1964), 51-78. 
[15] Green, A.E. and Rivlin, R.S., Multipolar continuum mechanics, Arch. Rational Mech. Anal., 17 (1964), 113-147.

[16] Fried, E. and Gurtin, M.E., Thermomechanics of the interface between a body and its environment, Continuum Mechanics and Thermodynamics, 19(5) (2007), 253-271.

[17] Flavin, J.N., Knops, R.J., Some spatial decay estimates in continuum dynamics. J. Elasticity, 17 (1987), 249-264.

[18] Flavin, J.N., Knops, R.J., Energy bounds in dynamical problems for a semiinfinite elastic beam. In: Eason, G., Ogden, R.W. (Eds.), Elasticity: Mathematical Methods and Applications, The Ian N. Sneddon 70th birthday volume. Ellis Horwood Limited, Chichester, pp. 101-112, 1990.

[19] Knops, R.J., Spatial decay estimates in the vibrating anisotropic elastic beam. In: Rionero, S. (Ed.), Waves and Stability in Continuum Media. World Scientific, Singapore, pp. 192-203, 1991.

[20] Chirita, S., Spatial decay estimates for solutions describing harmonic vibrations in a thermoelastic cylinder. J. Therm. Stresses, 18 (1995), 421436.

[21] Quintanilla, R., Straughan, B., A note on discontinuity waves in type III thermoelasticity. Proc. R. Soc. Lond. A, 460 (2004), 1169-1175.

Marin MARIN,

Department of Mathematics and Computer Science,

Transilvania University of Braşov,

Bdul Eroilor 29, 500036 Braşov, Romania.

Email: m.marin@unitbv.ro

Adina CHIRILĂ,

Department of Mathematics and Computer Science,

Transilvania University of Braşov,

Bdul Eroilor 29, 500036 Braşov, Romania.

Email: adina.chirila@unitbv.ro

Lavinia CODARCEA,

Department of Mathematics and Computer Science,

Transilvania University of Braşov,

Bdul Eroilor 29, 500036 Braşov, Romania.

Email: lavinia.codarcea.munteanu@unitbv.ro

Sorin VLASE,

Department of Mechanical Engineering,

Transilvania University of Braşov,

Str Politehnicii 1, 500024 Braşov, Romania.

Email: svlase@unitbv.ro 